\title{
Optic atrophy 1 mediates mitochondria remodeling and dopaminergic neurodegeneration linked to complex I deficiency
}

\author{
D Ramonet ${ }^{1,6}$, C Perier ${ }^{1,6}$, A Recasens $^{1}$, B Dehay ${ }^{1,7}$, J Bové ${ }^{1}$, V Costa $^{2,3}$, L Scorrano ${ }^{2,3}$ and M Vila ${ }^{*, 1,4,5}$
}

Mitochondrial complex I dysfunction has long been associated with Parkinson's disease (PD). Recent evidence suggests that mitochondrial involvement in PD may extend beyond a sole respiratory deficit and also include perturbations in mitochondrial fusion/fission or ultrastructure. Whether and how alterations in mitochondrial dynamics may relate to the known complex I defects in PD is unclear. Optic atrophy 1 (OPA1), a dynamin-related GTPase of the inner mitochondrial membrane, participates in mitochondrial fusion and apoptotic mitochondrial cristae remodeling. Here we show that complex I inhibition by parkinsonian neurotoxins leads to an oxidative-dependent disruption of OPA1 oligomeric complexes that normally keep mitochondrial cristae junctions tight. As a consequence, affected mitochondria exhibit major structural abnormalities, including cristae disintegration, loss of matrix density and swelling. These changes are not accompanied by mitochondrial fission but a mobilization of cytochrome $c$ from cristae to intermembrane space, thereby lowering the threshold for activation of mitochondria-dependent apoptosis by cell death agonists in compromised neurons. All these pathogenic changes, including mitochondrial structural remodeling and dopaminergic neurodegeneration, are abrogated by OPA1 overexpression, both in vitro and in vivo. Our results identify OPA1 as molecular link between complex I deficiency and alterations in mitochondrial dynamics machinery and point to OPA1 as a novel therapeutic target for complex I cytopathies, such as PD.

Cell Death and Differentiation (2013) 20, 77-85; doi:10.1038/cdd.2012.95; published online 3 August 2012

Reduced activity in mitochondrial complex I (NADH/ubiquinone oxidoreductase) impairs mitochondrial respiration and is associated with a wide spectrum of neurodegenerative diseases, including Parkinson's disease (PD). ${ }^{1}$ Reduced complex I activity has been found in autopsy brain tissues and platelets of patients affected with sporadic PD. ${ }^{1}$ In addition, complex I inhibitor 1-methyl-4-pheny-1,2,3,6tetrahydropyridine (MPTP) reproduces some of the clinical and neuropathological hallmarks of PD in monkeys and humans, including degeneration of dopaminergic neurons of the substantia nigra pars compacta $(\mathrm{SNpc}) .{ }^{1}$ In rodents, complex I inhibition with MPTP or rotenone leads to SNpc dopaminergic cell death through the activation of mitochondria-dependent apoptotic pathways. ${ }^{2-6}$ Growing evidence indicate, however, that the potential role of mitochondria in PD may extend well beyond a sole deficit in respiration. ${ }^{7}$ Genetic- and neurotoxic-based experimental systems related to PD have indeed unraveled perturbations in several aspects of mitochondria dynamics, including alterations in mitochondria fusion/fission balance, abnormalities in mitochondria morphology/structure or defects in mitochondria turnover. ${ }^{7}$ It is currently unknown whether and how these novel mechanisms by which mitochondria may also be linked to the pathogenesis of PD are actually related to the previously reported complex I defects occurring in this disease.

Optic atrophy 1 (OPA1) is a dynamin-related GTPase residing in the inner mitochondrial membrane (IMM) that participates in both the regulation of mitochondrial fusion and the pro-apoptotic remodeling of mitochondria. ${ }^{8,9}$ OPA1 promotes the IMM fusion whereas other GTPases, such as Mitofusins 1 and 2 (Mfn1, Mfn2), are involved in the fusion of the outer mitochondrial membrane (OMM) ${ }^{8}$ In addition, OPA1 has been shown to regulate mitochondria structural alterations occurring during apoptosis independently of its role in mitochondrial fusion. ${ }^{9}$ Biochemical analyses identified an

\footnotetext{
${ }^{1}$ Neurodegenerative Diseases Research Group, Vall d'Hebron Research Institute-CIBERNED, Barcelona, Spain; ${ }^{2}$ Dulbecco-Telethon Institute, Venetian Institute of Molecular Medicine, Padova, Italy; ${ }^{3}$ Department of Cell Physiology and Medicine, University of Geneva, Geneva, Switzerland; ${ }^{4}$ Department of Biochemistry and Molecular Biology, Autonomous University of Barcelona, Barcelona, Spain and ${ }^{5}$ Catalan Institution for Research and Advanced Studies (ICREA), Barcelona, Spain ${ }^{*}$ Corresponding author: M Vila, Neurodegenerative Diseases Research Group, Vall d'Hebron Research Institute, Mediterranean Building, Lab. 102, Pg. Vall d'Hebron 119-129, Barcelona 08035, Spain. Tel: +34 93489 4543; Fax: +34 93489 4015; E-mail: mvila@ir.vhebron.net

${ }^{6}$ These authors contributed equally to this work.

${ }^{7}$ Present address: CNRS-University of Bordeaux, Institut des Maladies Neurodégénératives, UMR 5293, Bordeaux, France

Keywords: Parkinson's disease; MPTP; rotenone; apoptosis

Abbreviations: AAV, adenoassociated viral; Drp1, dynamin-related protein 1; EDC, ethyl-3-(3-dimethylaminopropyl) carbodiimide; GFP, green fluorescent protein; IMM, inner mitochondrial membrane; IMS, mitochondria intermembrane space; Mfn1/2, Mitofusins 1 and 2; MPP ${ }^{+}, 1$-methyl-4-phenylpyridinium; MPTP, 1-methyl-4pheny-1,2,3,6-tetrahydropyridine; OMM, outer mitochondrial membrane; OPA1, Optic atrophy 1; PARL, presenilin-associated rhomboid-like protease; PD, Parkinson's disease; PINK1, PTEN-induced putative kinase-1; ROS, reactive oxygen species; SNpc, substantia nigra pars compacta; TH, tyrosine hydroxylase; TMPD, N,N,N', $N^{\prime}$ tetramethyl-p-phenylenediamine; TOM, translocase of the mitochondrial outer membrane; UT, untreated

Received 24.12.11; revised 28.5.12; accepted 20.6.12; Edited by R Youle; published online 03.8.12
} 
oligomeric OPA1 complex able to keep mitochondria cristae junctions tight in healthy mitochondria, thereby maintaining the bulk of cytochrome $c$ within cristae, where most mitochondrial respiration occurs. ${ }^{9}$ During apoptosis, this OPA1 complex is disrupted, leading to the widening of cristae junctions and the subsequent redistribution of cytochrome $c$ from cristae to the mitochondria intermembrane space (IMS). ${ }^{9}$ Once soluble in the IMS, cytochrome $c$ can be subsequently released to the cytosol by permeabilization of the OMM and initiate caspase-dependent cell death. Supporting an instrumental role for OPA1 in cell death, OPA1 knockdown in cultured cells results in mitochondrial morphological alterations, cytochrome $c$ release and apoptosis, whereas OPA1 overexpression protects against a range of pro-apoptotic stimuli. $^{10,11}$ In humans, mutations in OPA1 cause inherited neurodegenerative disorders, such as dominant optic atrophy, in which retinal ganglion cells degenerate. ${ }^{12,13}$ Given its key position at the crossroad between mitochondrial dynamics and cell death, we explored whether OPA1 may underlie mitochondrial alterations and dopaminergic neurodegeneration linked to PD-related complex I defects.

\section{Results}

OPA1 restores mitochondria morphological alterations linked to complex I inhibition. Diseases linked to defective mitochondrial function, including complex I cytopathies, are characterized by morphologically abnormal, swollen mitochondria with distorted cristae. ${ }^{14}$ Here we assessed whether OPA1 may underlie such mitochondrial structural abnormalities linked to complex I deficiency. Mitochondrial morphology was determined at ultrastructural level in SH-SY5Y neuroblastoma cells transfected with OPA1 or empty (control) vectors and treated with either saline or 1-methyl-4-phenylpyridinium (MPP ${ }^{+}$; i.e. MPTP's active metabolite). In salinetreated cells, whether transfected or not with OPA1, most mitochondria (95\% and $98 \%$, respectively) exhibited normal (class I) ultrastructure, with fairly dark, uniform matrix filled with densely packed, regularly distributed cristae (Figure 1a). Following $\mathrm{MPP}^{+}$intoxication, empty vector-transfected cells exhibited abundant ( $\sim 83 \%$ ) remodeled (class II) mitochondria with disrupted cristae, sometimes entirely lacking, and loss of matrix density (Figure 1a). Some of these abnormal
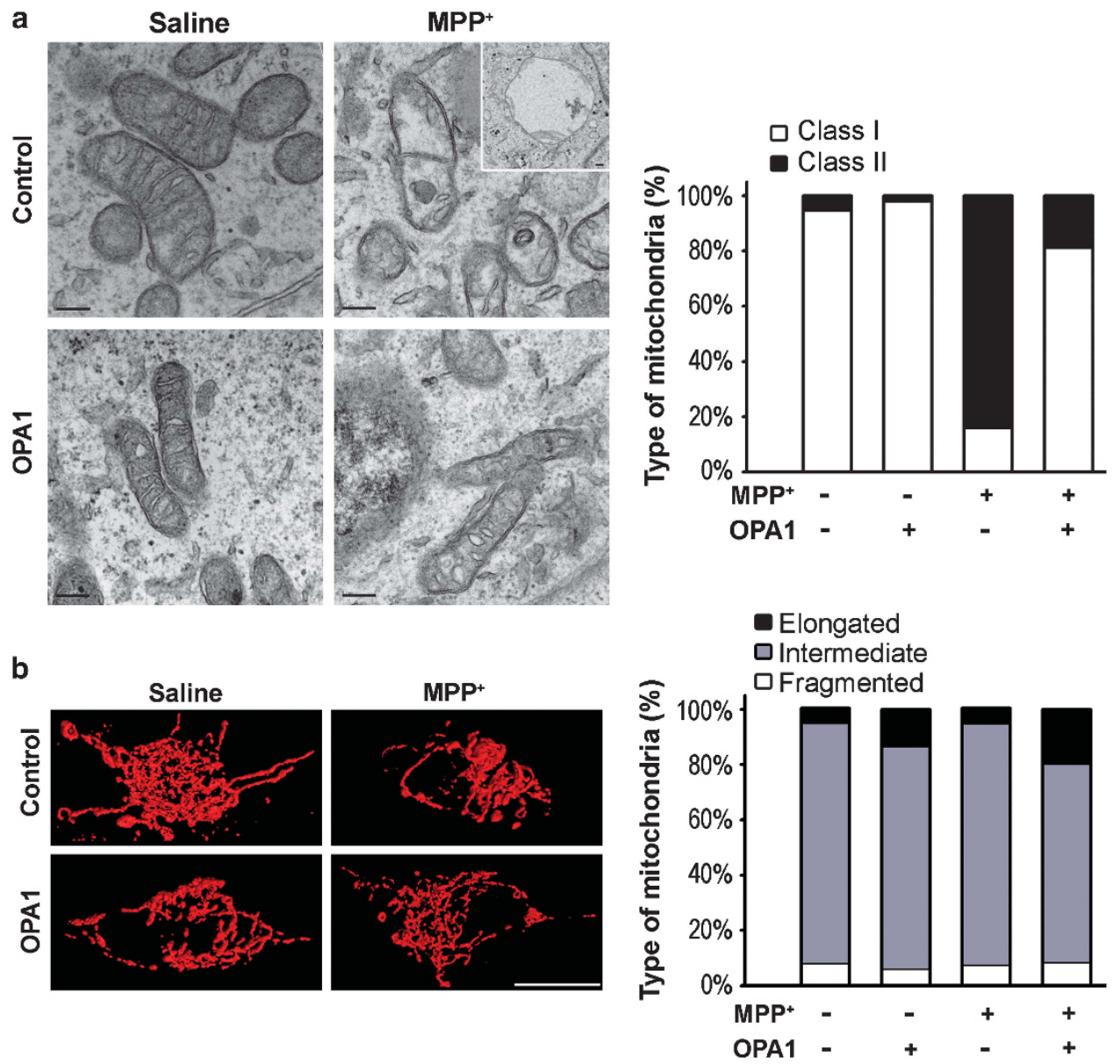

Figure 1 OPA1-dependent mitochondrial ultrastructural abnormalities, but not fission, following complex I inhibition. (a) Representative transmission electron microscopy images of the mitochondria in control and OPA1-overexpressing SH-SY5Y neuroblastoma cells treated with saline or MPP ${ }^{+}$. The percentage of normal (class I) and remodeled (class II) mitochondria was determined by analyzing a total of 293 mitochondria uniformly selected at random among the different genotype/treatment groups. (b) Representative images of mito-DsRed2-labeled mitochondria in control and OPA1-overexpressing SH-SY5Y neuroblastoma cells treated with saline or MPP ${ }^{+}$. Mitochondrial length was measured by morphometry and categorized as elongated $(>3 \mu \mathrm{m})$, intermediate $(0.5-3 \mu \mathrm{m})$ or fragmented $(<0.5 \mu \mathrm{m})$. A total of 1433 mito-DsRed2-labeled mitochondria were analyzed uniformly selected at random among the different genotype/treatment groups. MPP ${ }^{+}, 5 \mathrm{mM}$ for $24 \mathrm{~h}$. Scale bars, $0.2 \mu \mathrm{m}$ in (a), $10 \mu \mathrm{m}$ in (b) 
mitochondria appeared largely swollen and contained electron-dense inclusion bodies (Figure 1a, inset). OPA1 overexpression markedly reduced the amount of abnormal mitochondria (down to $\sim 19 \%$ ) in $\mathrm{MPP}^{+}$-treated cells (Figure 1a), thus indicating that mitochondrial structural abnormalities linked to complex I inhibition are modulated by OPA1. Quantitative analyses of (i) the mitochondrial area occupied by cristae and (ii) the electron density contributed by cristae within individual mitochondria further confirmed that $\mathrm{MPP}^{+}$induces a significant disruption of mitochondria cristae that can be prevented by OPA1 overexpression (Supplementary Figure 1).

We next determined whether mitochondrial morphological alterations caused by complex I inhibition were associated to changes in mitochondrial fusion/fission balance. Mitochondrial length was measured by morphometry in saline- or $\mathrm{MPP}^{+}$-treated SH-SY5Y cells co-transfected with the mitochondrial expression vector pDsRed2-Mito and either OPA1 or empty control vectors. On the basis of previous studies, ${ }^{15,16}$ mito-DsRed2-labeled mitochondria were binned into three different categories: mitochondria with a length of $>3 \mu \mathrm{m}$ were considered as elongated, those with a length between 0.5 and $3 \mu \mathrm{m}$ as intermediate and those smaller than $0.5 \mu \mathrm{m}$ as fragmented. In saline-treated, empty vector-transfected cells, $87 \%$ of mitochondria were of intermediate size, $8 \%$ appeared as fragmented and $5 \%$ as elongated (Figure $1 \mathrm{~b}$ ). Following $\mathrm{MPP}^{+}$intoxication, no significant changes in mitochondrial length categorization were detected, with $87 \%$ of mitochondria remaining at intermediate size, $5 \%$ as elongated and $7.5 \%$ as fragmented (Figure 1b). OPA1 overexpression increased the percentage of elongated mitochondria in both saline- and $\mathrm{MPP}^{+}$-treated cells (up to $13 \%$ and $19 \%$, respectively), although most mitochondria remained at intermediate size $(80 \%$ and $72 \%$, respectively) (Figure 1b). These results indicate that OPA1dependent mitochondrial structural abnormalities linked to complex I inhibition are not associated with significant mitochondrial fragmentation. Therefore, the above-reported effect of OPA1 at preventing $\mathrm{MPP}^{+}$-induced mitochondrial structural abnormalities cannot be attributed to an effect of OPA1 at counteracting mitochondrial fission in this pathological situation.
Complex I inhibition leads to oxidative-dependent OPA1 desoligomerization. In healthy mitochondria, the integrity of cristae is maintained by an oligomeric OPA 1 complex that, by clamping juxtaposed cristae membranes, keeps cristae junctions tight. $^{9}$ This oligomer, whose size is compatible with an OPA1 trimer $(\sim 290 \mathrm{kDa})$, corresponds to an heteromer formed by integral IMM and soluble IMS forms of OPA1, corresponding to long (L-OPA1, 100 kDa) and short (S-OPA1, $\sim 80 \mathrm{kDa}$ ) OPA1 isoforms, respectively. ${ }^{9}$ Given our observation of OPA1-dependent structural alterations in mitochondria cristae following complex I inhibition, we next determined whether OPA1 desoligomerization could account for such mitochondrial morphological defects in this pathological situation. As OPA1-containing complexes are chemically cross-linkable, we addressed this question by performing crosslinking experiments in isolated nonsynaptosomal mouse brain mitochondria. Untreated (UT) or $\mathrm{MPP}^{+}$treated mouse brain mitochondria were cross-linked with the zero-length crosslinker 1-ethyl-3-(3-dimethylaminopropyl) carbodiimide (EDC). In cross-linked UT brain mitochondria, a high-order OPA1 complex can be detected at $\sim 290 \mathrm{kDa}$ by immunoblot (Figure 2a). This OPA1 complex is absent in mitochondria whose cristae had been mechanically distended by osmotic swelling (Figure 2a). Following complex I inhibition with $\mathrm{MPP}^{+}$, brain mitochondria exhibited a dosedependent reduction in oligomeric OPA1 (Figures 2a and b), which was accompanied by parallel increases in non-oligomeric OPA1 isoforms (Supplementary Figure 2a). Similar results were observed in native gels without crosslinking (Supplementary Figure 3), and when treating brain mitochondria with another complex I inhibitor, rotenone (Supplementary Figures $2 \mathrm{~b}$ and 4 ). In contrast to OPA1, $\mathrm{MPP}^{+}$does not destabilize other mitochondrial protein complexes such as complex I (Supplementary Figure 5) or the translocase of the mitochondrial outer membrane (TOM complex) (Supplementary Figure 6).

Because complex I blockade is associated with increased mitochondrial production of reactive oxygen species (ROS), ,17 we next explored whether OPA1 desoligomerization caused by complex I inhibition may be secondary to increased mitochondrial oxidative damage. Supporting the latter, incubation of isolated brain mitochondria with the free-radical scavenger
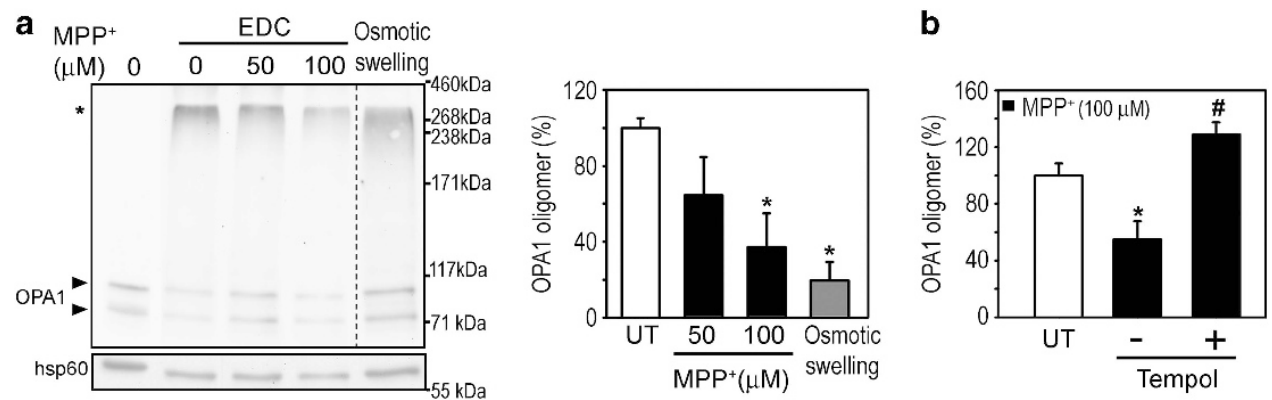

Figure 2 Oxidative-mediated OPA1 desoligomerization following complex I inhibition. (a) Isolated nonsynaptosomal mouse brain mitochondria treated with different doses of $\mathrm{MPP}^{+}$or osmotically swollen for 15 min were incubated with $1 \mathrm{mM}(E D C)$ for 30 min followed by centrifugation. Proteins in the pellet were separated by SDS-PAGE and immunoblotted using anti-OPA1 antibodies. The asterisk indicates OPA1 oligomer; arrowheads indicate non-oligomeric OPA1. Histograms represent average quantification of OPA1 oligomers \pm S.E.M. from at least three independent experiments. (b) Isolated nonsynaptosomal mouse brain mitochondria were treated, processed and quantified as in $\mathbf{a}$, in the presence or absence of tempol $(500 \mu \mathrm{M}$ for $15 \mathrm{~min})$. In (a and $\mathbf{b}),{ }^{*} P<0.05$, compared with untreated mitochondria; in $\mathbf{b}$, ${ }^{\#} P<0.05$, compared with $\mathrm{MPP}^{+}$-treated, tempol-free mitochondria 
$\mathbf{a}$

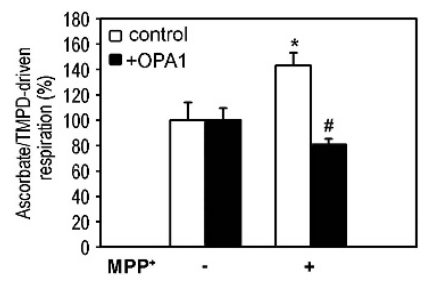

b

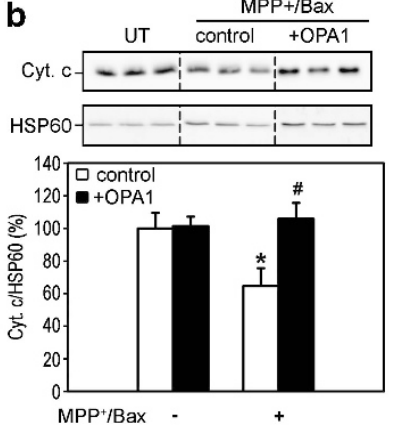

C

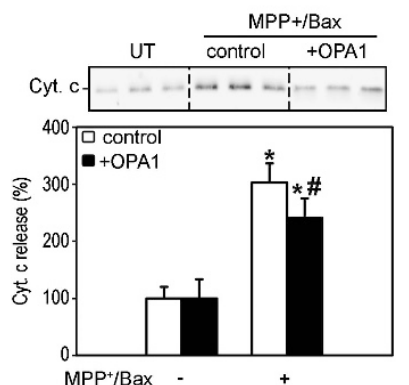

Figure 3 OPA1-dependent mobilization of cytochrome $c$ following complex I inhibition. (a) Mitochondrial ascorbate/TMPD-driven respiration ratio in isolated nonsynaptosomal mouse brain mitochondria treated with $\mathrm{MPP}^{+}(500 \mu \mathrm{M}$ for $5 \mathrm{~min})$ preincubated with either recombinant OPA1 or reticulocyte lysate without OPA1 (control) for $25 \mathrm{~min}$. (b and $\mathbf{c}$ ) Isolated nonsynaptosomal mouse brain mitochondria were treated with a combination of MPP ${ }^{+}(100 \mu \mathrm{M})$ and recombinant Bax (100 nM) for 15 min, in the presence of either recombinant OPA1 or reticulocyte lysate without OPA1 (control). After centrifugation, the amount of cytochrome $c$ in supernatant (b) and pellet (c) was determined by immunoblot. Histograms represent average \pm S.E.M. from at least three independent experiments. ${ }^{*} P<0.05$, compared with untreated mitochondria; ${ }^{\#} P<0.05$, compared with $\mathrm{MPP}^{+}$-treated control mitochondria

and superoxide dismutase mimetic compound tempol completely abrogated $\mathrm{MPP}^{+}$-induced OPA1 desoligomerization (Figure 2b). Overall, our results indicate that complex I inhibition leads to an oxidative-mediated disruption of the OPA1-containing oligomeric complexes that normally keep mitochondria cristae junctions in check.

OPA1 disruption linked to complex I inhibition results in cytochrome $c$ mobilization. By tightening cristae junctions, oligomeric OPA1 helps maintaining the bulk of cytochrome $c$ within cristae in healthy mitochondria. ${ }^{9}$ Therefore, we next determined whether OPA1 desoligomerization caused by complex I blockade is associated with a mobilization of cytochrome $c$ from cristae to IMS. Given the different redox potential and accessibility of membrane bound and free cytochrome $c$, these two pools of cytochrome $c$ can be specifically reduced by ascorbate and membrane-permeant $N, N, N^{\prime}, N^{\prime}$-tetramethyl-p-phenylenediamine (TMPD), the former being able to reduce only soluble cytochrome $c$ while the latter can reach all cytochrome $c$. The ratio of ascorbateover TMPD-driven oxygen consumption (asc/TMPD) thus provides an estimate of the pool of free cytochrome $c$ in the IMS relative to the total mitochondrial cytochrome $c^{4,18}$ In isolated mouse brain mitochondria, inhibition of complex I with $\mathrm{MPP}^{+}$leads to increased asc/TMPD-driven respiration, indicating a mobilization of cytochrome $c$ from cristae stores toward the IMS (Figure 3a). ${ }^{4}$ Supporting a role for OPA1 disruption on $\mathrm{MPP}^{+}$-induced cristae-to-IMS cytochrome $c$ mobilization, the latter was markedly attenuated in isolated brain mitochondria preincubated with recombinant OPA1 (Figure 3a), which readily internalizes into mitochondrial membranes (Supplementary Figures $7 a$ and 8 ).

Once soluble in the IMS, cytochrome $c$ can be released to the cytosol following permeabilization of the OMM by proapoptotic members of the $\mathrm{Bcl}-2$ family. In the context of dopaminergic neurodegeneration linked to complex I inhibition, OMM permeabilization is mediated by cell death agonist Bax. ${ }^{2,4,5}$ Reflecting the requirement for both cristae remodeling and OMM permeabilization for cytochrome $c$ release linked to complex I blockade, neither complex I inhibition by itself nor the sole permeabilization of the OMM with recombinant Bax are able to trigger overt cytochrome $c$ release from isolated brain mitochondria. ${ }^{4}$ In contrast, the combination of complex I inhibition with recombinant Bax results in a marked release of cytochrome $c$ from these mitochondria (Figures $3 \mathrm{~b}$ and $\mathrm{c}$ ). ${ }^{4}$ Consistent with the abovereported effect of OPA1 at preventing $\mathrm{MPP}^{+}$-induced cytochrome $c$ mobilization from cristae to IMS (Figure 3a), the release of cytochrome $c$ induced by $\mathrm{Bax}$ in $\mathrm{MPP}^{+}$-treated brain mitochondria was markedly attenuated by the addition of recombinant OPA1 (Figures $3 b$ and $c$ ). The beneficial effects of OPA1 at preventing cytochrome $c$ mobilization and Bax-mediated cytochrome $c$ release in $\mathrm{MPP}^{+}$-treated mitochondria were not due to an impaired ability of MPP ${ }^{+}$ to inhibit complex I and increase ROS production in the presence of recombinant OPA1 (Supplementary Figure 9). Taken together, our results indicate that OPA1 desoligomerization linked to complex I inhibition increases the releasable soluble pool of cytochrome $c$ in the IMS, thereby sensitizing affected mitochondria to PD-related pro-cell death agonists, such as Bax.

OPA1 attenuates cytochrome $c$ release and cell death linked to complex I inhibition in vitro. We next assessed the pathogenic significance of OPA1 disruption and mitochondrial remodeling caused by complex I inhibition. SH-SY5Y cells co-transfected with pDsRed2-Mito and either OPA1 or empty control vectors were treated with saline or complex I inhibitors $\left(\mathrm{MPP}^{+}\right.$or rotenone) and subsequently immunostained for cytochrome $c$. In salinetreated cells, whether transfected or not with OPA1, most cytochrome $c(78 \%$ and $82 \%$, respectively) colocalized with mito-DsRed2-labeled mitochondria, reflecting the typical mitochondrial localization of cytochrome $c$ in healthy mitochondria (Figures $4 \mathrm{a}$ and b). Following $\mathrm{MPP}^{+}$intoxication, empty vector-transfected cells exhibited a markedly reduced colocalization of cytochrome $c$ with mito-DsRed2 (Figures 4a and $b$ ), indicative of cytochrome $c$ being released from affected mitochondria in these cells. MPP ${ }^{+}$-induced cytochrome $c$ release was associated with cell death, as determined by flow cytometry after propidium iodide staining (Figure 4c). OPA1 overexpression markedly attenuated $\mathrm{MPP}^{+}$-induced 
a
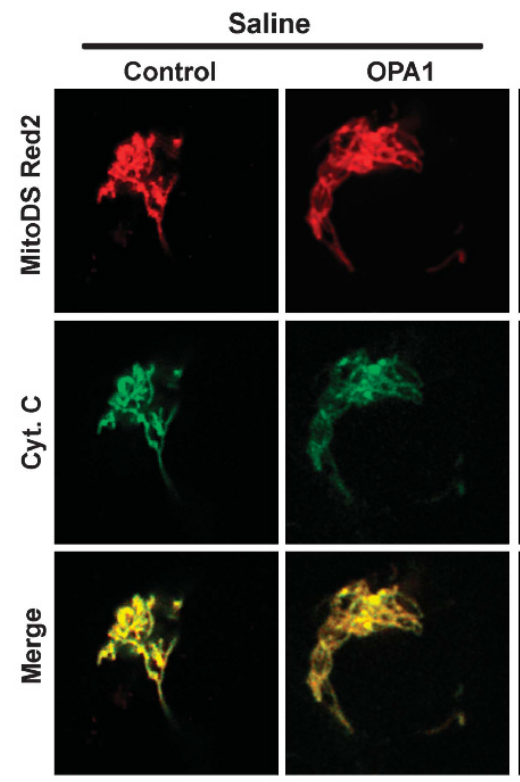

\section{b}

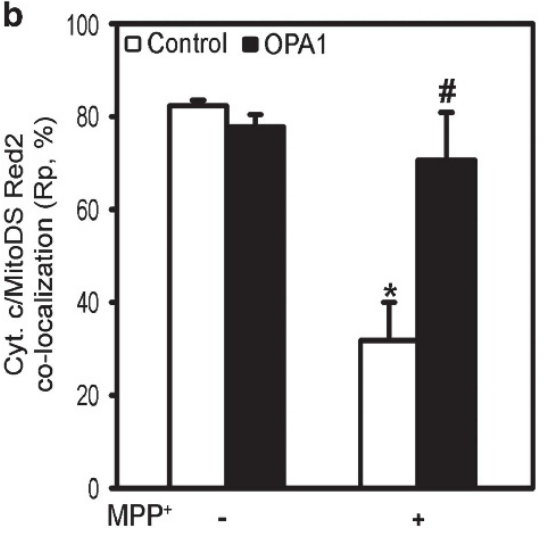

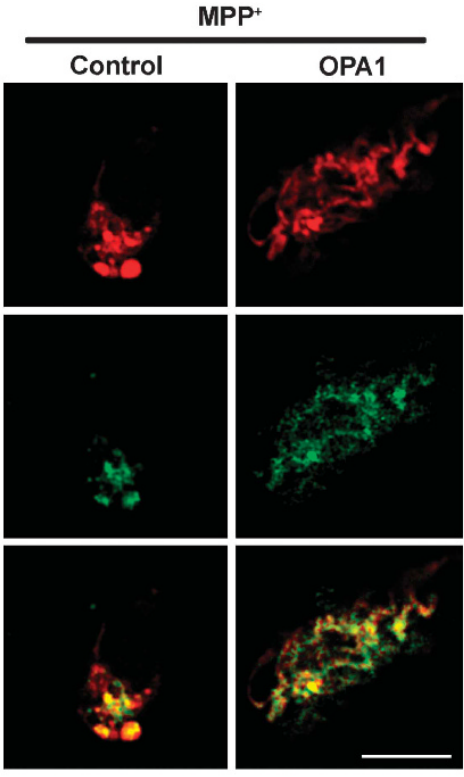

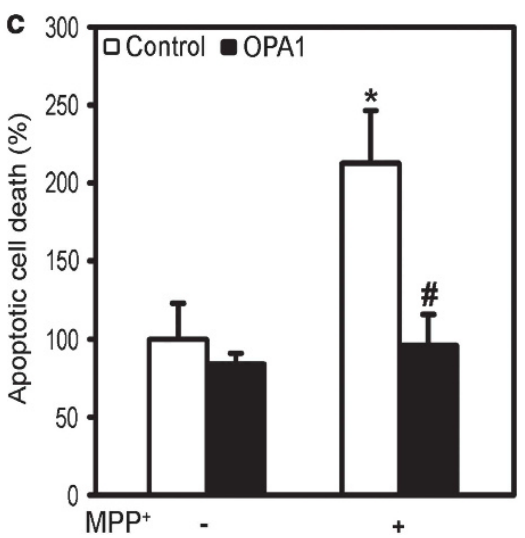

Figure 4 OPA1 overexpression attenuates cytochrome $c$ release and cell death linked to complex I inhibition. (a) Representative images of cytochrome $c$ subcellular distribution (in green; red corresponds to mito-DsRed2-labeled mitochondria) in control (empty vector) and OPA1-overexpressing SH-SY5Y neuroblastoma cells treated with saline or $\mathrm{MPP}^{+}$. (b) Pearson's colocalization coefficients (Rp) between cytochrome $c$ and mito-DsRed2-labeled mitochondria in the different experimental groups. At least three representative fields containing an average of 45 cells/field were analyzed per group. (c) Cell death determined by flow cytometry after propidium iodide staining in control (empty vector) and OPA1-overexpressing SH-SY5Y cells treated with saline or MPP ${ }^{+}$. Histograms represent average \pm S.E.M. from at least three independent experiments. MPP ${ }^{+}, 5 \mathrm{mM}$ for $24 \mathrm{~h}$; ${ }^{*} P<0.05$, compared with untreated cells; ${ }^{\sharp} P<0.05$, compared with MPP ${ }^{+}$-treated control (empty vector) cells. Scale bar, $10 \mu \mathrm{m}$

cytochrome $c$ release (Figures $4 a$ and b) and cell death (Figure 4c). Similar results were obtained when treating these cells with rotenone (Supplementary Figure 10). These results indicate that OPA1 disruption has a major pathogenic role in neurodegeneration linked to complex I blockade in vitro.

OPA1 attenuates dopaminergic nigrostriatal denervation linked to complex I inhibition in vivo. To determine the relevance of our in vitro results to an in vivo situation, we assessed OPA1 immunoblot levels by SDS-PAGE in ventral midbrain samples from mice treated with either saline or MPTP (30 mg/kg per day for five consecutive days). In saline-injected animals, OPA1 was detected as a double band corresponding to integral IMM (L-OPA1, 100 kDa) and soluble IMS (S-OPA1, 80 kDa) OPA1 isoforms (Figure 5a). Both IMM and IMS OPA1 isoforms were markedly decreased in the ventral midbrain of MPTP-treated mice, compared with saline-injected animals (Figure 5a). Decreased OPA1 levels occurred early following MPTP (i.e. $24 \mathrm{~h}$ after the last MPTP injection), thereby preceding dopaminergic cell death in this model, which occurs between $48 \mathrm{~h}$ and 4 days post MPTP. ${ }^{2,4,5}$ These results concur with the observed OPA1 desoligomerization and increased apoptotic cell death in $\mathrm{MPP}^{+}$-treated cells, as levels of IMS/IMM OPA1 are crucial for the formation of the OPA1 oligomer to protect from apoptosis ${ }^{19}$ and to prevent cristae remodeling. ${ }^{9}$ To further confirm whether OPA1 decreased levels has an instrumental role in MPTP-induced neurodegeneration, we overexpressed OPA1 in the SNpc of salineand MPTP-treated mice by means of adenoassociated viral (AAV) vectors. Mice received single unilateral stereotaxic injections of AAV-OPA1 in the SNpc. Control mice received identical injections of an AAV vector encoding for green fluorescent protein (GFP; AAV-GFP). Three weeks after viral vector delivery, ventral midbrain samples ipsilateral 
a
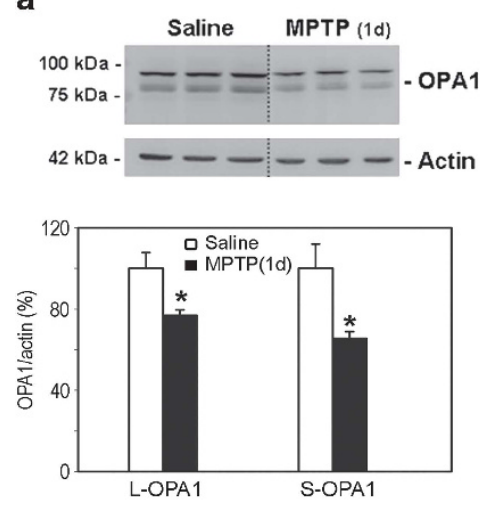

b
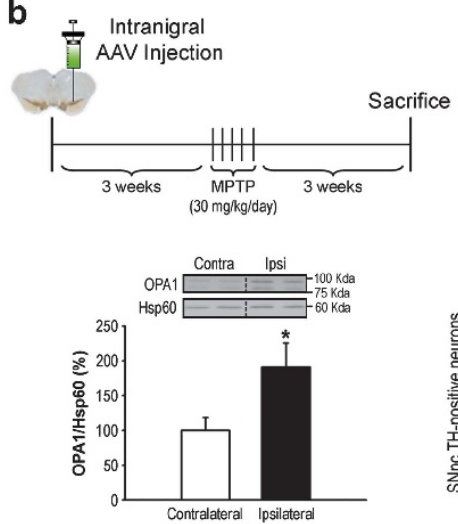

c
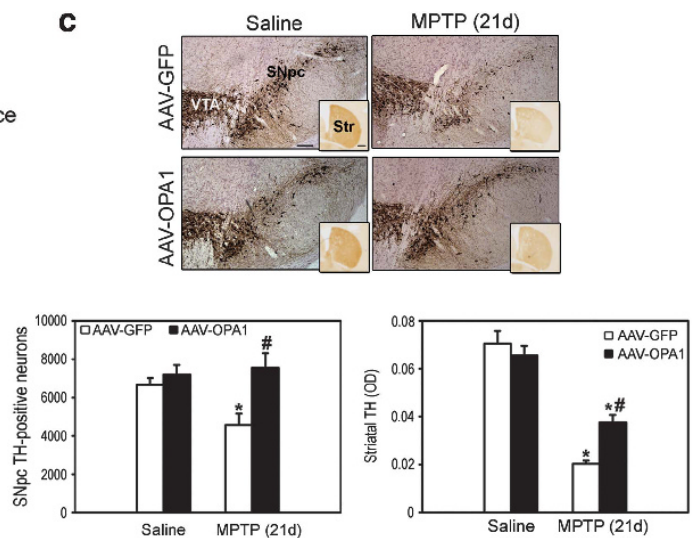

Figure 5 OPA1 overexpression attenuates dopaminergic nigrostriatal denervation in MPTP-intoxicated mice. (a) OPA1 immunoblot levels (L-OPA1, 100 kDa; S-OPA1, $\sim 80 \mathrm{kDa}$ ) by SDS-PAGE in ventral midbrain samples from mice treated with either saline or MPTP ( $30 \mathrm{mg} / \mathrm{kg}$ per day for 5 consecutive days) and killed $24 \mathrm{~h}$ ( 1 day) after the last saline or MPTP injection. (b) Schematic representation of the experimental design used for AAV-OPA1 injections in MPTP-treated mice (top). OPA1 immunoblot levels in mouse ventral midbrain samples ipsilateral and contralateral to AAV-OPA1 injections (bottom). (c) Representative photomicrographs of TH-immunostained SNpc (brown; thionin in purple) and striatum (Str, inset) from saline- and MPTP-treated mice, overexpressing GFP or OPA1, at day 21 post MPTP (top). Stereological cell counts of SNpc THimmunoreactive neurons (left) and optical densitometry of striatal TH immunoreactivity (right) in the different experimental groups of animals at day 21 post MPTP (bottom). Histograms represent average \pm S.E.M. $\left(n=5-8\right.$ animals per group); ${ }^{*} P<0.05$ compared with saline-injected mice; ${ }^{\#} P<0.05$ compared with MPTP-treated GFP-expressing mice; Scale bar, $500 \mathrm{mM}$

to AAV-OPA1 injections exhibited an approximately twofold increase in total OPA1 levels by immunoblot, compared with the contralateral (non-injected) side (Figure 5b). Mitochondria isolated from AAV-OPA1-injected ventral midbrains confirmed that overexpressed OPA1 was internalized into mitochondria (Supplementary Figure 7b). OPA1- and GFP-overexpressing mice were treated with either saline or MPTP and the integrity of the nigrostriatal dopaminergic system was assessed in these animals at day 21 after the last MPTP injection, once the dopaminergic lesion is stabilized using this particular regimen of MPTP intoxication. $^{20}$ In AAV-GFP-injected mice, MPTP killed $\sim 30 \%$ of SNpc dopaminergic neurons, as determined by stereological cell counts of SNpc tyrosine hydroxylase (TH)positive cells (Figure $5 \mathrm{c}$ ), and produced a $\sim 70 \%$ depletion of striatal dopaminergic terminals, as assessed by optical densitometry of striatal TH-positive fibers (Figure $5 \mathrm{c}$ ). In contrast, OPA1-overexpressing mice exhibited a marked attenuation of MPTP-induced nigrostriatal dopaminergic denervation, both at the level of SNpc dopaminergic cell bodies and striatal dopaminergic terminals (Figure 5c). These results indicate that OPA1 has an instrumental role for dopaminergic neurodegeneration linked to complex I inhibition in vivo and demonstrate the feasibility and neuroprotective potential of targeting OPA1 in this pathological situation.

\section{Discussion}

Here we identified OPA1 as a major regulator of mitochondrial morphological abnormalities and dopaminergic neurodegeneration linked to complex I deficiency, both in vitro and in vivo. Complex I inhibition leads to the disruption of OPA1 oligomeric complexes that normally maintain cristae structure in healthy mitochondria. As a consequence, affected mitochondria exhibit major structural abnormalities, such as reduced number of cristae, occasionally entirely lacking, loss of matrix density and swelling. Remarkably, these mitochondrial changes can be reversed by increasing OPA1 levels. Relevant to PD, similar defects in mitochondrial morphology, including cristae disintegration, have been observed in cytoplasmic hybrid (cybrid) cells repopulated with mitochondria from PD patients ${ }^{14}$ and in substantia nigra postmortem PD samples. ${ }^{21,22}$ In addition, PD-causing pathogenic mutations in Parkin (an E3 ubiquitin ligase) and PINK1 (PTENinduced putative kinase-1, a mitochondrially targeted kinase) induce similar mitochondrial morphological alterations, as observed in Drosophila melanogaster Pink1 or park (the $D$. melanogaster homologue of the parkin-encoding gene $P A R K 2)$ mutants ${ }^{23-27}$ or in primary fibroblasts derived from PD patients carrying two different PINK1 mutations. ${ }^{28}$ Supporting a potential role for of OPA1 in PINK1/parkindependent mitochondrial abnormalities, OPA1 has been identified as a PD-interacting gene in genome-wide screen analyses for modifiers of PD genes in Drosophila Pink1/park mutants. $^{29}$

In our study, mitochondrial structural abnormalities linked to complex I inhibition were not associated with significant mitochondrial fragmentation. Although earlier reports have indicated the occurrence of mitochondrial fission following intoxication by parkinsonian neurotoxins, ${ }^{30-32}$ recent studies failed to detect mitochondrial fragmentation in $\mathrm{MPP}^{+}$-treated murine mesencephalic dopaminergic primary neurons. ${ }^{33}$ Although the reasons for such apparent discrepancies are unknown, mitochondrial fission has not been demonstrated so far in PD, although the latter may be hindered by current technical limitations. In our experimental system, cytochrome $c$ release and cell death linked to complex I deficiency correlated with mitochondrial structural remodeling rather than mitochondrial fission. Supporting our observation, mitochondrial fragmentation is uncoupled from cytochrome $c$ release and cell death induced by Bax/Bak activation in HeLa cells. ${ }^{34}$ In addition, enforced mitochondrial elongation by expression of pro-fusion proteins Mfn1 or Mnf2 fails to protect 
against huntingtin-mediated or NMDA-induced cell death, respectively, while preservation of cristae structure by OPA1 overexpression is able to attenuate cell death in both pathological situations. ${ }^{16,35}$ Furthermore, OPA1 overexpression efficiently protects cells lacking Mfn1, which is essential for OPA1-mediated fusion, and cells doubly deficient for Mfn1 and Mfn2, in which fusion is completely abolished, ${ }^{9}$ thereby indicating that OPA1 exerts its anti-apoptotic effects independently from mitochondrial fusion. Taken together, these results point to cristae remodeling, rather than mitochondrial fission, as an essential component of mitochondriadependent apoptosis. In apparent contradiction with the latter assertion, inhibition of pro-fission dynamin-related protein 1 (Drp1) protects cells against a variety of pro-apoptotic stimuli, including parkinsonian neurotoxins ${ }^{30-32}$ and parkin/PINK1 PD-causing mutations. ${ }^{36,37}$ Reconciling these results, some studies have shown that Drp1 not only promotes mitochondrial fragmentation but can also induce OPA1-dependent cristae remodeling. ${ }^{35,38,39}$ Therefore, the reported antiapoptotic effects linked to Drp1 inhibition may actually be exerted by preventing cristae remodeling and not mitochondrial fission.

OPA1 oligomers are presumably produced by heterodimerization of a soluble IMS form of OPA1 with integral IMM OPA1 isoforms. ${ }^{9}$ In mammalian mitochondria, the formation of soluble IMS OPA1 depends, at least in part, on the inner membrane presenilin-associated rhomboid-like (PARL) protease. ${ }^{9,19}$ Accordingly, PARL-deficient mitochondria exhibit decreased OPA1 oligomerization and are more prone to apoptosis. ${ }^{9,19}$ Remarkably, a missense mutation in the PARL gene, leading to defective PARL's activity, has been recently identified in two patients with PD. ${ }^{40}$ Although it remains to be determined whether $P D$-linked mutant PARL affects OPA1 oligomerization, this observation further reinforces a potential link between OPA1 and PD. In our study, OPA1 desoligomerization linked to complex I inhibition was found to be dependent on increased mitochondrial ROS production, suggesting that OPA1 may be a target of mitochondrial oxidative stress. In this context, we have previously shown that complex I inhibition leads to the peroxidation of the IMM phospholipid cardiolipin, to which cytochrome $c$ is normally attached, resulting in the disruption of the normal binding of cytochrome $c$ to the IMM. ${ }^{4}$ Combined with the results presented here, it appears that cristae-to-IMS mobilization of cytochrome $c$ following complex I inhibition involves the detachment of cytochrome $c$ from the IMM and the widening of cristae junctions, both phenomena being secondary to mitochondrially-driven oxidative attack (to cardiolipin and OPA1, respectively) (Figure 6). Further supporting a pathogenic role for mitochondria-derived ROS in PD-related neurodegeneration, transgenic mice overexpressing human catalase specifically targeted to the mitochondria exhibit an attenuation of MPTP-induced mitochondrial ROS and reduced dopaminergic neurodegeneration. ${ }^{17}$

OPA1-dependent cristae remodeling leads to the redistribution of cytochrome $c$ from cristae to the IMS, from where it can be subsequently released to the cytosol following OMM permeabilization and initiate caspase-dependent cell death. OMM permeabilization linked to complex I inhibition has been shown to be mediated by Bax. ${ }^{2,4,5}$ MPTP-intoxicated mice
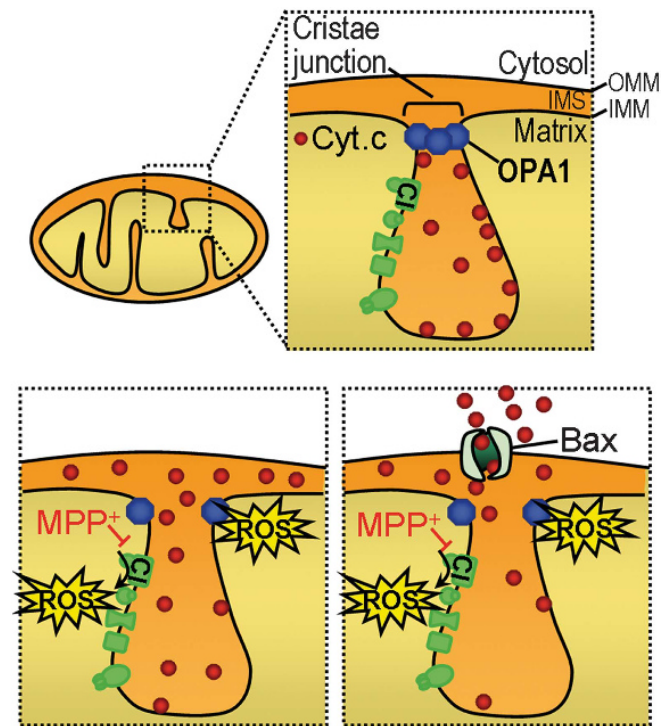

Figure 6 Schematic representation of the proposed mechanism of OPA1dependent mobilization of cytochrome $c$ linked to complex I inhibition (see text for details). Cl, complex I; Cyt. c, cytochrome $c$

exhibit a time-dependent, region-specific cytochrome $c$ release followed by activation of caspase- 9 , caspase- 3 and apoptotic dopaminergic cell death. ${ }^{4}$ All these events, including dopaminergic neurodegeneration, coincide with Bax mitochondrial translocation and are prevented by genetic ablation of Bax. $^{2,4,5}$ In contrast, other pro-apoptotic cell death agonists, such as Bid or Bak, which are known for cooperating with Bax to initiate mitochondrial-dependent apoptosis in response to the ligation of cell-surface death receptors, are dispensable for MPTP-induced dopaminergic neurodegeneration (Supplementary Figure 11). Remarkably, the extent of neuroprotection provided by OPA1 overexpression in MPTP-intoxicated mice is very similar to that obtained by the genetic ablation of Bax in these animals, ${ }^{2}$ thus being compatible with both approaches acting within the same molecular pathway. In addition, both approaches not only preserve SNpc dopaminergic cell bodies following MPTP intoxication but also attenuate striatal dopaminergic terminal loss. ${ }^{2}$ The latter is of particular therapeutic importance in the context of PD, because the cardinal motor symptoms of the disease are actually attributed to the loss of striatal dopamine fibers. ${ }^{1}$

Intriguingly, loss of OPA1 (and decreased mitochondrial fusion) was shown to rescue flight muscle degeneration, cell death and mitochondrial abnormalities caused by PD-linked pink1/parkin mutations in Drosophila. ${ }^{41,42}$ This effect has been attributed to a restoration of mitochondrial fission and an increased ability to degrade dysfunctional, fragmented mitochondria through mitophagy. ${ }^{41-43}$ In contrast to the observations in Drosophila, the results presented here in human cells and mice indicate instead that the loss of Opa1 severely compromises neurons by sensitizing these cells for the proapoptotic release of cytochrome $c$. This apparent discrepancy may be attributed to the fact that, in contrast to mammalian cells, OMM permeabilization and cytochrome $c$ release are not consistently required for insect cell apoptosis. ${ }^{44}$

In summary, our study identifies OPA1 as a molecular link between complex I deficiency and alterations in mitochondrial 
dynamics machinery, both of which have been independently associated to PD. According to our study, dopaminergic cell death linked to complex I deficiency correlates with OPA1dependent mitochondria structural remodeling, but not mitochondrial fission, rendering compromised neurons more prone to degenerate. More importantly, we provide the first demonstration of a neuroprotective effect obtained by manipulation of molecular components of the mitochondrial dynamics machinery, such as OPA1, in an in vivo situation. These results point to OPA1 as a potential disease-modifying therapeutic target for complex I cytopathies, such as PD.

\begin{abstract}
Materials and Methods
Materials. For immunoblotting, the primary antibodies used were OPA1 (BD biosciences, San Jose, CA, USA; 612606), cytochrome $c$ (BD biosciences), Hsp60 (Santa Cruz Biotechnology, Santa Cruz, CA, USA), NDUFA9 (MitoSciences, Eugene, OR, USA) and TOM20 (Abcam, Cambridge, UK). For immunostaining, primary antibodies were cytochrome $c$ (Abcam, ab6400) and TH (Calbiochem, Darmstadt, Germany). Complex I inhibitors $\mathrm{MPP}^{+}$and rotenone were obtained from Sigma-Aldrich. pDsRed2-Mito plasmid was obtained from Clontech (Mountain View, CA, USA). pCDNA3.1-OPA-1 plasmids was developed as described. ${ }^{9}$ Recombinant oligomeric Bax was obtained from Abnova (Taipei City, Taiwan). AAV vectors of serotype $2 / 6$ encoding for OPA1 were produced at the UPV-CBATEG (Autonomous University of Barcelona, UAB, Spain).
\end{abstract}

Cell culture and treatments. SH-SY5Y human dopaminergic neuroblastoma cells were obtained from ATCC and maintained at $37^{\circ} \mathrm{C}$ in $5 \% \mathrm{CO}_{2}$ in MEM/ Ham's F12 medium, supplemented with $10 \%$ fetal calf serum and $1 \%$ penicillin/ streptomycin, until use. Transfections were performed with lipofectamide 2000 (Invitrogen, Paisley, UK) following the manufacturer recommendations. For drug treatments, cells were grown to $70-80 \%$ confluency.

Electron microscopy. After the indicated treatments, cells were detached, pelleted and fixed in $2 \%$ glutaraldehyde for $2 \mathrm{~h}$ at $4{ }^{\circ} \mathrm{C}$, then postfixed in $1 \%$ osmium tetroxide for $1 \mathrm{~h}$ at $4{ }^{\circ} \mathrm{C}$. The samples were dehydrated, embedded in plastic and cut into in 70-nm sections for microscopy. Sections were subsequently poststained with 5\% uranyl acetate. Samples were viewed with a JEOL JEM 1010 electron microscope (Tokyo, Japan). Classification of mitochondrial morphological subtypes (Class I/II) was performed as previously reported. ${ }^{18}$

Mitochondrial length. SH-SY5Y cells were stably transfected with mitochondrial expression vector pDsRed2-Mito. After the indicated treatments, cells were fixed with prewarmed $3.7 \%$ formaldehyde, and high resolution $3 D$ confocal stacks were obtained. Image reconstructions were performed with ImageJ, and 3D morphometry measures were performed with Imaris.

Cytochrome $\mathrm{c} / \mathrm{mitochondria}$ colocalization assays. Following the indicated drug treatments, SH-SY5Y cells stably transfected with pDsRed2-Mito were immunostained with a sheep anti-cytochrome $c$ antibody (Abcam; 1:1000) and the corresponding anti-sheep FITC-labeled secondary antibody. 3D stacks were obtained in an Olympus FV1000 confocal microscope (Olympus, Tokyo, Japan) and the Pearson's correlation coefficient (Rp) between cytochrome $c$ and mito-DsRed2-labeled mitochondria were calculated for each plane. At least three representative fields containing an average of 45 cells/field were used per experimental condition.

Cell death assay. Cell death was quantified by flow cytometry after propidium iodide staining. At selected time points after treatment, cells were detached and collected by centrifugation, resuspended and fixed in $70 \%$ ethanol at $-20^{\circ} \mathrm{C} \mathrm{O}$ $\mathrm{N}$. After centrifugation, the pellet was stained for $30 \mathrm{~min}$ with $50 \mu \mathrm{g} / \mathrm{ml}$ propidium iodide staining solution ( $3.8 \mathrm{mM}$ sodium citrate, $50 \mu \mathrm{g} / \mathrm{ml}$ RNaseA in PBS). Fluorescence measurements and analyses were performed using FACSCalibur flow cytometer (BD biosciences).

Isolation of brain mitochondria, cytochrome $c$ release assays, polarography. Nonsynaptosomal brain mitochondria was obtained as previously reported. ${ }^{4}$ Briefly, brains from C57BL/6 mice were homogenized in isolation buffer (225 mM mannitol, $75 \mathrm{mM}$ sucrose, $1 \mathrm{mM}$ EGTA, $20 \mathrm{mM}$ HEPES and $0.1 \%$ fat-free BSA) using a motorized Dounce homogenizer with eight up-anddown strokes. The homogenate was centrifuged at $1000 \times g$ for $10 \mathrm{~min}$, and the resulting supernatant was layered onto a gradient of Ficoll and centrifuged at $79000 \times g$ for $30 \mathrm{~min}$. The mitochondrial pellet was resuspended in isolation buffer. Protein concentrations were determined by the bicinchoninic assay method (Pierce Chemical Co., Rockford, IL, USA) with BSA as a standard protein. For cytochrome $c$ release experiments, $300 \mu \mathrm{g}$ of isolated brain mitochondria were incubated for $15 \mathrm{~min}$ at RT in constant agitation with $\mathrm{MPP}^{+}(100 \mu \mathrm{M})$ and recombinant oligomeric Bax $(100 \mathrm{nM}$; Abnova), and pelleted at $12000 \times g$ for $15 \mathrm{~min}$ at $4{ }^{\circ} \mathrm{C}$. The percentage of cytochrome $c$ release was estimated by assessing the intensities of the immunoblot bands in both particulate and soluble mitochondrial fractions, as previously described. ${ }^{4}$ Oxygen consumption was monitored using a Clark-type electrode (Hansatech Instruments, PP Systems, Haverhill, MA, USA), as described. ${ }^{4}$

Ascorbate/TMPD assay. These experiments were performed as described. ${ }^{4,18}$ Briefly, $1 \mathrm{mg} / \mathrm{ml}$ mitochondria was incubated in sucrose buffer (0.2 M sucrose/10 mM trisphosphate-4-morpholinepropanesulfonic acid, $\mathrm{pH} 7.4$ / $1 \mathrm{mM} \mathrm{Pi} / 5 \mathrm{mM}$ glutamate $/ 2.5 \mathrm{mM}$ malate $/ 10 \mu \mathrm{M}$ EGTA-phosphate Tris, $\mathrm{pH}$ 7.4) and treated as indicated. After the indicated time, $400 \mathrm{pmol}$ carbonyl cyanide 3chlorophenylhydrazone and $1 \mathrm{nmol}$ antimycin A per $\mathrm{mg}$ of protein were added, and the reaction was transferred to a Clark-type oxygen electrode chamber. Final volume was $1 \mathrm{ml}$ at $25^{\circ} \mathrm{C}$. After $2 \mathrm{~min}, 6 \mathrm{mM}$ ascorbate was added, followed by $300 \mu \mathrm{M}$ TMPD 3 min thereafter. The ascorbate-driven oxygen consumption rate over the total TMPD-driven rate was plotted as a percentage of the ratio in the UT mitochondria.

Recombinant OPA1 production. OPA1 recombinant protein was synthesized in vitro using the TnT quick-coupled transcription/translation system from Promega. Recombinant biotinylated OPA-1 was obtained by adding Transcend tRNA to the TnT reaction mix (Promega, Madison, WI, USA). In all experiments, reticulocyte lysate without Opa1 was used as control.

Animals and treatments. Eight-to 10-week-old C57BL/6 mice received one i.p. injection of MPTP-HCl per day ( $30 \mathrm{mg} / \mathrm{kg}$ of free base; Sigma-Aldrich, St. Louis, MO, USA) for 5 consecutive days and were killed at the indicated time points after the last MPTP injection; control mice received saline injections only.

Assessment of OPA1 contents in isolated ventral midbrain mitochondria by flow cytometry. Isolated brain mitochondria from dissected ventral midbrains were incubated with isolation buffer $1 \%$ BSA containing a 1:50 dilution of mouse monoclonal anti-OPA1 antibody (BD biosciences). Alexa 647 anti-mouse $(1: 500)$ was used as secondary antibody. Flow cytometry was performed in a FACSCalibur flow cytometer (BD biosciences). OPA-1 signal was recorded on FL4-H.

AAV vector production and sterotaxic delivery. AAV vector of serotype $2 / 6$ encoding for OPA1 were produced at the UPV-CBATEG (Autonomous University of Barcelona, UAB, Spain). Control AAV 2/6 vectors encoding for GFP were kindly provided by Dr. M Monfar (UPV-CBATEG). Viral titers were $1.6 \times 10^{12}$ viral particles/ $\mu$ l for AVV-OPA1 and $1.2 \times 10^{12}$ for AAVEGFP. One microliter of viral suspension was stereotaxically delivered to the substantia nigra of 6-week-old C57BL/6 mice ( $-2.9 \mathrm{AP},-1.3 \mathrm{~L}$ and $-4.2 \mathrm{DV}$ ) at a flow rate of $0.2 \mu \mathrm{l} / \mathrm{min}$. At day 21 post AAV injections, animals were killed or subsequently treated with saline or MPTP, as indicated.

Quantitative morphology. Twenty-one days after the last MPTP injection, mice were killed, and their brains were removed and processed for immunohistochemistry using a polyclonal anti-TH (1:1000; Calbiochem). The total number of TH-positive SNpc was estimated by stereology using the optical fractionator method. ${ }^{2}$ Optical densitometry of striatal TH-positive terminals was determined using Scion Image software, as described. ${ }^{17}$

Statistical analysis. All values are expressed as the mean \pm S.E.M. Differences among means were analyzed by using one- or two-way ANOVA. When ANOVA showed significant differences, pair-wise comparisons between means were tested by Student-Newman-Keuls post hoc testing. In all analyses, the null hypothesis was rejected at the 0.05 level. 


\section{Conflict of Interest}

The authors declare no conflict of interest.

Acknowledgements. We thank A Parent and E Pérez for their technical assistance, $T$ Lindsten (University of Pennsylvania, USA) for providing Bak-deficient mice and A Strasser (The Walter and Eliza Hall Institute of Medical Research, Australia) for providing Bid-deficient mice. This work was supported by European Commission's Marie Curie Excellence Grant and Marie Curie International Reintegration Grant (MV), Fundació la Caixa, Spain (MV), FIS-ISCIII, Spain (MV and CP), MICINN, Spain (MV), Ramón y Cajal Program (MICINN, to CP) and Miguel Servet Program (FIS-ISCIII, to JB)

1. Dauer W, Przedborski S. Parkinson's disease: mechanisms and models. Neuron 2003; 39 889-909.

2. Vila $M$, Jackson-Lewis $V$, Vukosavic $S$, Djaldetti $R$, Liberatore $G$, Offen $D$ et al. Bax ablation prevents dopaminergic neurodegeneration in the 1-methyl- 4-phenyl-1,2,3,6 tetrahydropyridine mouse model of Parkinson's disease. Proc Natl Acad Sci USA 2001 98: 2837-2842.

3. Vila M, Przedborski S. Targeting programmed cell death in neurodegenerative diseases Nat Rev Neurosci 2003; 4: 365-375.

4. Perier C, Tieu K, Guegan C, Caspersen C, Jackson-Lewis V, Carelli V et al. Complex deficiency primes Bax-dependent neuronal apoptosis through mitochondrial oxidative damage. Proc Natl Acad Sci USA 2005; 102: 19126-19131.

5. Perier C, Bove J, Wu DC, Dehay B, Choi DK, Jackson-Lewis $V$ et al. Two molecular pathways initiate mitochondria-dependent dopaminergic neurodegeneration in experimental Parkinson's disease. Proc Natl Acad Sci USA 2007; 104: 8161-8166.

6. Sherer TB, Betarbet R, Testa CM, Seo BB, Richardson JR, Kim JH et al. Mechanism of toxicity in rotenone models of Parkinson's disease. J Neurosci 2003; 23: $10756-10764$

7. Schon EA, Przedborski S. Mitochondria: the next (neurode)generation. Neuron 2011; 70 1033-1053.

8. Chen $\mathrm{H}$, Chan DC. Mitochondrial dynamics - fusion, fission, movement, and mitophagy in neurodegenerative diseases. Hum Mol Genet 2009; 18: R169-R176.

9. Frezza C, Cipolat S, Martins DB, Micaroni M, Beznoussenko GV, Rudka T et al. OPA1 controls apoptotic cristae remodeling independently from mitochondrial fusion. Cell 2006; 126: 177-189.

10. Lee YJ, Jeong SY, Karbowski M, Smith CL, Youle RJ. Roles of the mammalian mitochondrial fission and fusion mediators Fis1, Drp1, and Opa1 in apoptosis. Mol Biol Cell 2004; 15: 5001-5011.

11. Olichon A, Baricault L, Gas N, Guillou E, Valette A, Belenguer P et al. Loss of OPA1 perturbates the mitochondrial inner membrane structure and integrity, leading to cytochrome c release and apoptosis. J Biol Chem 2003; 278: 7743-7746.

12. Delettre C, Lenaers G, Griffoin JM, Gigarel N, Lorenzo C, Belenguer $P$ et al. Nuclear gene OPA1, encoding a mitochondrial dynamin-related protein, is mutated in dominant optic atrophy. Nat Genet 2000; 26: 207-210.

13. Zuchner S, Mersiyanova IV, Muglia M, Bissar-Tadmouri N, Rochelle J, Dadali EL et al. Mutations in the mitochondrial GTPase mitofusin 2 cause Charcot-Marie-Tooth neuropathy type 2A. Nat Genet 2004; 36: 449-451.

14. Trimmer PA, Swerdlow RH, Parks JK, Keeney P, Bennett JP Jr, Miller SW et al. Abnorma mitochondrial morphology in sporadic Parkinson's and Alzheimer's disease cybrid cel lines. Exp Neurol 2000; 162: 37-50.

15. Nakamura K, Nemani VM, Azarbal F, Skibinski G, Levy JM, Egami K et al. Direct membrane association drives mitochondrial fission by the Parkinson disease-associated protein alpha-synuclein. J Biol Chem 2011; 286: 20710-20726.

16. Jahani-Asl A, Pilon-Larose K, Xu W, MacLaurin JG, Park DS, McBride HM et al. The mitochondrial inner membrane GTPase, optic atrophy 1 (Opa1), restores mitochondria morphology and promotes neuronal survival following excitotoxicity. J Biol Chem 2011 286: 4772-4782.

17. Perier C, Bove J, Dehay B, Jackson-Lewis V, Rabinovitch PS, Przedborski S et al Apoptosis-inducing factor deficiency sensitizes dopaminergic neurons to parkinsonian neurotoxins. Ann Neurol 2010; 68: 184-192.

18. Scorrano L, Ashiya M, Buttle K, Weiler S, Oakes SA, Mannella CA et al. A distinct pathway remodels mitochondrial cristae and mobilizes cytochrome c during apoptosis. Developmental Cell 2002; 2: 55-67.

19. Cipolat S, Rudka T, Hartmann D, Costa V, Serneels L, Craessaerts K et al. Mitochondrial rhomboid PARL regulates cytochrome $\mathrm{c}$ release during apoptosis via OPA1-dependent cristae remodeling. Cell 2006; 126: 163-175.
20. Jackson-Lewis V, Przedborski S. Protocol for the MPTP mouse model of Parkinson's disease. Nat Protoc 2007; 2: 141-151.

21. Anglade P, Vyas S, Javoy-Agid F, Herrero MT, Michel PP, Marquez J et al. Apoptosis and autophagy in nigral neurons of patients with Parkinson's disease. Histol Histopathol 1997; 12: $25-31$.

22. Sekiya S, Tanaka M, Hayashi S, Oyanagi S. Light- and electron-microscopic studies of intracytoplasmic acidophilic granules in the human locus ceruleus and substantia nigra. Acta Neuropathol 1982; 56: 78-80.

23. Clark IE, Dodson MW, Jiang $\mathrm{C}, \mathrm{Cao} \mathrm{JH}$, Huh JR, Seol JH et al. Drosophila pink1 is required for mitochondrial function and interacts genetically with parkin. Nature 2006; 441: 1162-1166.

24. Park J, Lee SB, Lee S, Kim Y, Song S, Kim S et al. Mitochondrial dysfunction in Drosophila PINK1 mutants is complemented by parkin. Nature 2006; 441 1157-1161.

25. Yang Y, Gehrke S, Imai Y, Huang Z, Ouyang Y, Wang JW et al. Mitochondrial pathology and muscle and dopaminergic neuron degeneration caused by inactivation of Drosophila Pink1 is rescued by Parkin. Proc Natl Acad Sci USA 2006; 103 : 10793-10798

26. Greene JC, Whitworth AJ, Kuo I, Andrews LA, Feany MB, Pallanck LJ. Mitochondrial pathology and apoptotic muscle degeneration in Drosophila parkin mutants. Proc Natl Acad Sci USA 2003; 100: 4078-4083.

27. Wang C, Lu R, Ouyang X, Ho MW, Chia W, Yu F et al. Drosophila overexpressing parkin R275W mutant exhibits dopaminergic neuron degeneration and mitochondrial abnormalities. J Neurosci 2007; 27: 8563-8570.

28. Exner N, Treske B, Paquet D, Holmstrom K, Schiesling C, Gispert S et al. Loss-of-function of human PINK1 results in mitochondrial pathology and can be rescued by parkin. J Neurosci 2007; 27: 12413-12418.

29. Fernandes C, Rao Y. Genome-wide screen for modifiers of Parkinson's disease genes in Drosophila. Mol Brain 2011; 4: 17.

30. Barsoum MJ, Yuan H, Gerencser AA, Liot G, Kushnareva Y, Graber S et al. Nitric oxideinduced mitochondrial fission is regulated by dynamin-related GTPases in neurons. EMBO J 2006; 25: 3900-3911.

31. Meuer K, Suppanz IE, Lingor P, Planchamp V, Goricke B, Fichtner $L$ et al. Cyclindependent kinase 5 is an upstream regulator of mitochondrial fission during neuronal apoptosis. Cell Death Differ 2007; 14: 651-661.

32. Gomez-Lazaro M, Bonekamp NA, Galindo MF, Jordan J, Schrader M. 6-Hydroxydopamine (6-OHDA) induces Drp1-dependent mitochondrial fragmentation in SH-SY5Y cells. Free Radic Biol Med 2008; 44: 1960-1969.

33. Kim-Han JS, Antenor-Dorsey JA, O'Malley KL. The Parkinsonian mimetic, MPP + specifically impairs mitochondrial transport in dopamine axons. J Neurosci 2011; 31: 7212-7221.

34. Sheridan C, Delivani P, Cullen SP, Martin SJ. Bax- or Bak-induced mitochondrial fission can be uncoupled from cytochrome $C$ release. Mol Cell 2008; 31: 570-585.

35. Costa V, Giacomello M, Hudec R, Lopreiato R, Ermak G, Lim D et al. Mitochondrial fission and cristae disruption increase the response of cell models of Huntington's disease to apoptotic stimuli. EMBO Mol Med 2010; 2: 490-503.

36. Lutz AK, Exner N, Fett ME, Schlehe JS, Kloos K, Lammermann K et al. Loss of parkin or PINK1 function increases Drp1-dependent mitochondrial fragmentation. J Biol Chem 2009; 284: 22938-22951

37. Cui M, Tang X, Christian WV, Yoon Y, Tieu K. Perturbations in mitochondrial dynamics induced by human mutant PINK1 can be rescued by the mitochondrial division inhibitor mdivi-1. J Biol Chem 2010; 285: 11740-11752.

38. Germain M, Mathai JP, McBride HM, Shore GC. Endoplasmic reticulum BIK initiates DRP1-regulated remodelling of mitochondrial cristae during apoptosis. EMBO J 2005; 24 : $1546-1556$

39. Mopert K, Hajek P, Frank S, Chen C, Kaufmann J, Santel A. Loss of Drp1 function alters OPA1 processing and changes mitochondrial membrane organization. Exp Cell Res 2009; 315: 2165-2180.

40. Shi G, Lee JR, Grimes DA, Racacho L, Ye D, Yang H et al. Functional alteration of PARL contributes to mitochondrial dysregulation in Parkinson's disease. Hum Mol Genet 2011; 20: $1966-1974$

41. Deng $\mathrm{H}$, Dodson MW, Huang $\mathrm{H}$, Guo $\mathrm{M}$. The Parkinson's disease genes pink1 and parkin promote mitochondrial fission and/or inhibit fusion in Drosophila. Proc Natl Acad Sci USA 2008; 105: 14503-14508.

42. Poole AC, Thomas RE, Andrews LA, McBride HM, Whitworth AJ, Pallanck LJ. The PINK1/Parkin pathway regulates mitochondrial morphology. Proc Natl Acad Sci USA 2008; 105: 1638-1643.

43. Narendra DP, Jin SM, Tanaka A, Suen DF, Gautier CA, Shen J et al. PINK1 is selectively stabilized on impaired mitochondria to activate Parkin. PLOS Biol 2010; 8: e1000298.

44. Martinou JC, Youle RJ. Mitochondria in apoptosis: Bcl-2 family members and mitochondrial dynamics. Dev Cell 2011; 21: 92-101. 